\title{
Incidental finding of aberrant subclavian artery during computed tomography scan
}

Yusof $\mathrm{MM}^{1}$, Shamsudin $\mathrm{S}^{2}$

'Mubarak Mohd Yusof, ${ }^{1}$ Sharini Shamsudin; Radiologists, Diagnostic Imaging Department, Tengku Ampuan Afzan Hospital, Jalan Tanah Putih, 25100 Kuantan, Pahang, Malaysia.

\section{ABSTRACT}

Two cases of aberrant subclavian arteries were detected incidentally during computed tomography scan of the thorax for other medical conditions. The patients did not have weight loss or dysphagia lusoria. The origin and course of the aberrant subclavian arteries are related to the anomaly of the aortic arch. The variations of aberrant right subclavian artery with left aortic arch and aberrant left subclavian artery with right aortic arch on computed tomography are discussed.

Key words: aberrant subclavian artery, computed tomography, dysphagia lusoria, left aortic arch, right aortic arch.

\section{INTRODUCTION}

\begin{abstract}
A $\mathrm{n}$ aberrant subclavian artery indicates an abnormal origin of the subclavian artery from the aortic arch. The right subclavian artery normally arises from the innominate artery. The left subclavian artery arises directly from the aortic arch after the left common carotid artery. In cases of aberrant subclavian artery, they originate as the last artery branching out from the aortic arch. Their position and course are different and might cause symptoms to the patient. The knowledge about these anatomical variants is important in order to identify the anomalies and to differentiate from other mediastinal vital structures.
\end{abstract}

\section{CASE REPORT}

\section{CASE 1}

A 64-year old man had gastrectomy two years ago for gastrointestinal tumour of the stomach. He was asymptomatic and came for contrast enhanced computed tomography (CECT) scan of the thorax and abdomen. The CECT scan revealed no evidence of recurrence or metastasis. He had normal left-sided aortic arch with an aberrant right subclavian artery (ARSA). The ARSA coursed posterior to the trachea and oesophagus

Address for correspondence

Mubarak Mohd Yusof

Radiologist

Diagnostic Imaging Department, Tengku Ampuan Afzan Hospital, Jalan Tanah Putih, 25100 Kuantan, Pahang, Malaysia

E-mail: adibawazif@yahoo.com with no compression of these structures (Figure 1).

\section{CASE 2}

A 69-year old man came for computed tomography pulmonary angiography (CTPA) to look for pulmonary embolism. He was admitted for shortness of breath for the past two days. He was incidentally found to have right-sided aortic arch with aberrant left subclavian artery (ALSA). The aorta had irregular lumen due to atheromatous plaque formation. The ALSA was posterior to the trachea and oesophagus and no compression of these structures were seen (Figure 2). There was no filling defect in the pulmonary arteries to suggest embolism.

\section{DISCUSSION}

An aberrant subclavian artery refers to a rare congenital variant of the subclavian artery origin in relation to the position of the aortic arch. The incidence is reportedly $0.05 \%$ for ALSA with right aortic arch and $0.5 \%$ to $2.0 \%$ for ARSA with left aortic arch'1. The left aortic arch with ARSA is the most common aortic arch anomaly involving the subclavian artery ${ }^{2,3}$.

The aberrant arteries and the aortic arch can be identified during CECT scan of the thorax. With the injection of intravascular contrast media, these arteries are easily differentiated from other structures such as oesophagus, lymph nodes and veins. Furthermore, with multiplanar reformatting (MPR), maximum intensity projection (MIP) and volume rendering (VR) of the axial images, the entire course of the arteries can be adequately assessed ${ }^{4,5}$. 


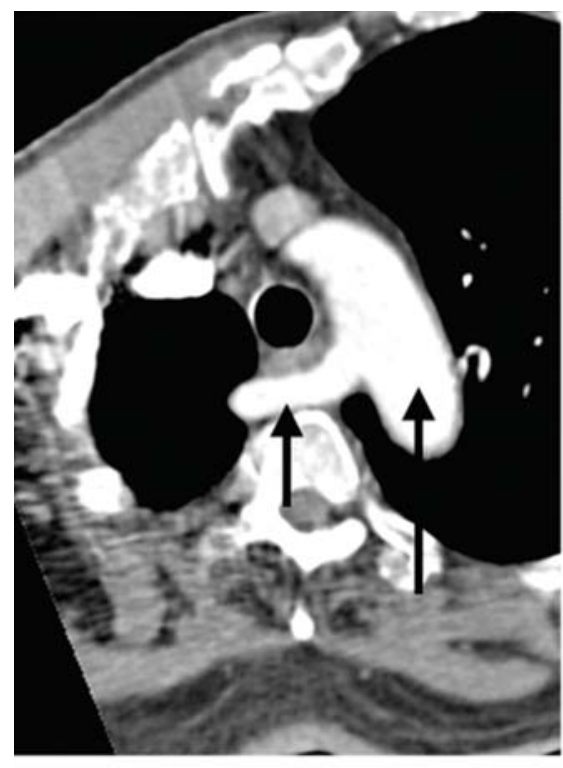

(a)

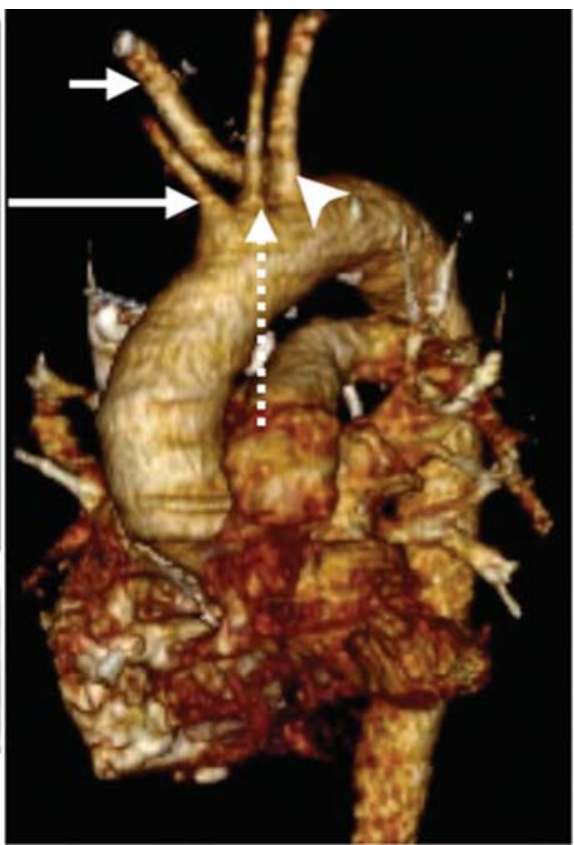

(b)

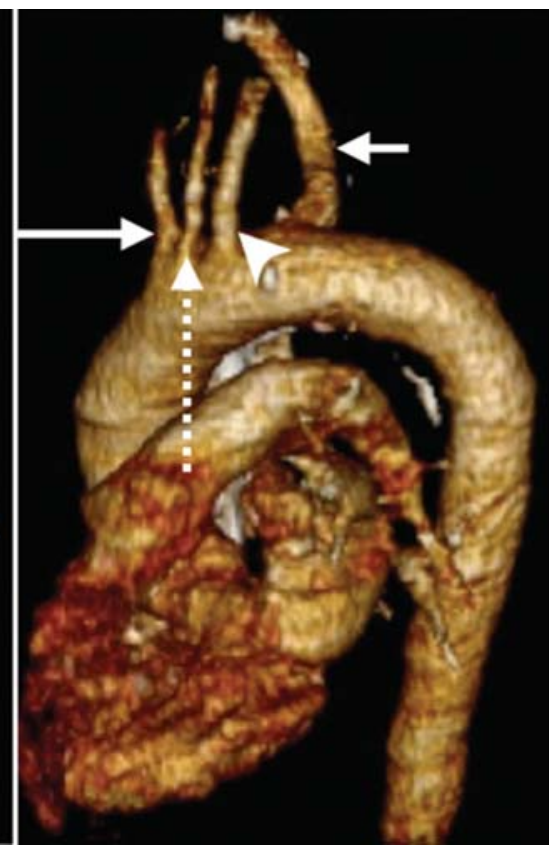

(c)

Figure 1: Left aortic arch with aberrant right subclavian artery (ARSA). (a) Axial CECT image shows left aortic arch (long arrow) and ARSA (short arrow) posterior to the trachea and oesophagus. Volume rendering images (b) and (c) show the ARSA (short arrow) arises from the aortic arch after the right common carotid (long arrow), left common carotid (dashed arrow) and left subclavian (arrowhead) arteries.

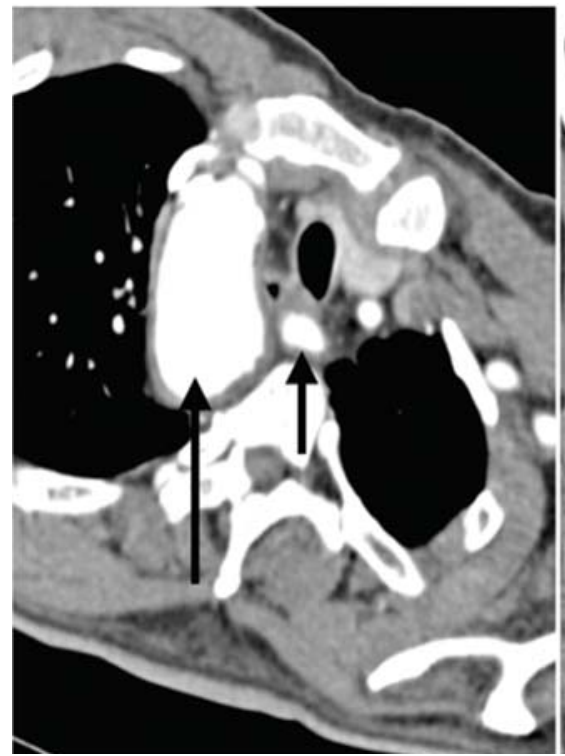

(a)

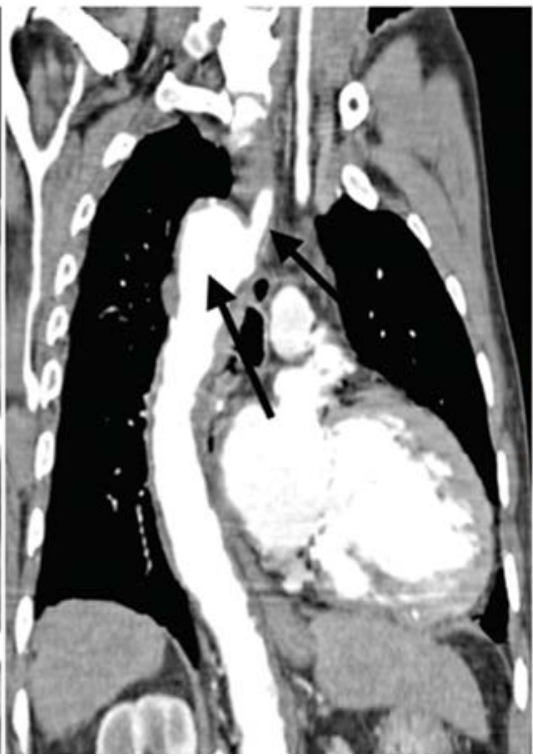

(b)

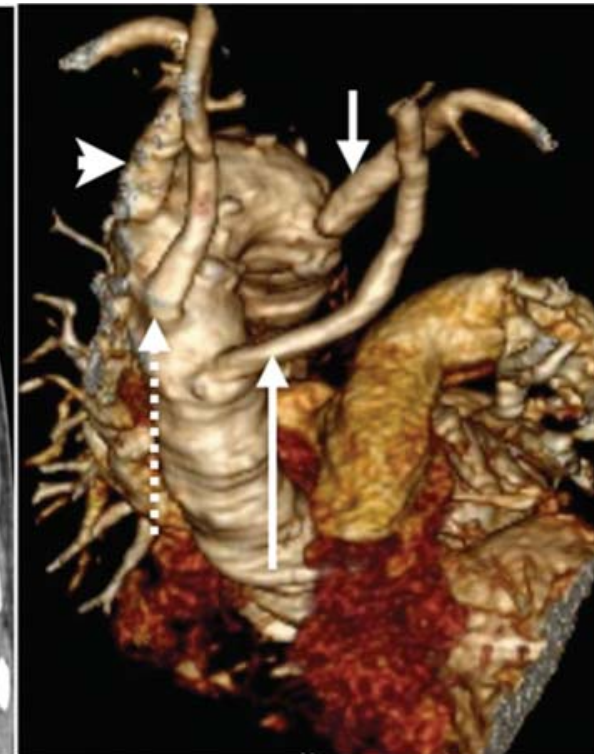

(c)

Figure 2: Right aortic arch with aberrant left subclavian artery (ALSA). CECT images in axial (a) and coronal reformatted (b) show right aortic arch (long arrow) and ALSA (short arrow). The descending aorta courses on the right side. (c) Volume rendering image shows the ALSA arises from the right aortic arch after the left common carotid (long arrow), right common carotid (dashed arrow) and right subclavian (arrowhead) arteries.

The aberrant arteries arise as the last arteries branching out from the aortic arch. The ALSA arises from the right aortic arch after the left common carotid, right common carotid and right subclavian arteries ${ }^{3}$. A few authors have described this branching pattern of arteries as Type 2 right aortic $\operatorname{arch}^{6-8}$. In left aortic arch, the ARSA arises 
after the right common carotid, left common carotid and left subclavian arteries ${ }^{3}$. The aberrant artery usually courses posterior to the oesophagus and less commonly anterior to the trachea or oesophagus ${ }^{5}$.

Embryologically, the aberrant subclavian arteries results from the developmental anomaly of the aortic arch. There is abnormal regression of the respective fourth aortic arch and interruption between the common carotid and subclavian artery ${ }^{9,10}$. The aberrant subclavian artery usually has dilation of the proximal portion adjacent to its origin from the aortic $\mathrm{arch}^{3}$. It is termed as Kommerell's diverticulum, lusoria diverticulum, remnant diverticulum or lusoria root and it was first described by Burckhard Friedrich Kommerell in 1936 in the case of left aortic arch with ARSA ${ }^{11}$.

The individuals with aberrant subclavian arteries are usually asymptomatic ${ }^{4,6,12-14}$. As in our two cases, CECT thorax was done for other medical conditions. The symptoms are usually due to atherosclerotic changes of the anomalous vessels, dissection, aneurysm with

\section{REFERENCES}

1. Yang MH, Weng ZC, Weng YG, Chang HH. A rightsided aortic arch with Kommerell's diverticulum of the aberrant left subclavian artery presenting with syncope. J Chin Med Assoc. 2009 May;72(5):275-7.

2. Walker TG, Geller SC. Aberrant right subclavian artery with a large diverticulum of Kommerell: a potential for misdiagnosis. Am J Roentgenol. 1987 Sep;149(3):477-8.

3. Türkvatan A, Büyükbayraktar FG, Olçer T, Cumhur T. Multidetector computed tomographic angiography of aberrant subclavian arteries. Vasc Med. 2009 Feb;14(1):5-11.

4. Türkvatan A, Büyükbayraktar FG, Olçer $T$, Cumhur T. Congenital anomalies of the aortic arch: evaluation with the use of multidetector computed tomography. Korean J Radiol. 2009 MarApr;10(2):176-84.

5. Onbas O, Kantarci $M$, Koplay $M$, Olgun $H$, Alper $\mathrm{F}$, Aydinli B, et al. Congenital anomalies of the aorta and vena cava: 16-detector-row CT imaging findings. DiagnIntervRadiol. 2008 Sep;14(3):163-71.

6. Cina CS, Arena GO, Bruin G, Clase CM. Kommerell's diverticulum and aneurysmal right-sided aortic arch: a case report and review of the literature. J Vasc Surg. 2000 Dec;32(6):1208-14. compression of adjacent structures causing dysphagia (dysphagia lusoria) and dyspnea ${ }^{3,6}$. The CECT scan examination is able to demonstrate these changes sufficiently.

A vascular ring can be found in aberrant subclavian artery. However, a vascular ring in left aortic arch with ARSA is rare $^{3}$. The vascular ring is formed by the left aortic arch with ARSA, right pulmonary artery and right ductusarteriosus. A complete vascular ring is formed by the right aortic arch with ALSA, left ductus arteriosus and left pulmonary artery. In both types of aberrant subclavian arteries, the vascular ring is generally too loose to compress on adjacent structures and produce symptoms $s^{3,14}$.

\section{CONCLUSION}

Though aberrant subclavian arteries are rare anomalies of the aortic arch, the knowledge of aortic arch variants is important when interpreting CECT scan of the thorax. CECT scan is a non-invasive method and has the ability to perform image reformatting to identify the anomalies.

7. Garti IJ, Aygen MM, Vidne B, Levy MJ. Right aortic arch with mirror- image branching causing vascular ring. A new classification of the right aortic arch patterns. Br J Radiol. 1973 Feb;46(542):115-9.

8. Knight L, Edwards JE. Right aortic arch: types and associated cardiac anomalies. Circulation. 1974 Nov;50(5):1047-51.

9. Tsutsumi M, Ueno Y, Kazekawa K, Tanaka A, Nomoto Y. Aberrant Right Subclavian Artery. Three Case Reports. Neurol Med Chir (Tokyo). 2002;42(9):396-8.

10. Salanitri J. MR angiography of aberrant left subclavian artery arising from right-sided thoracic aortic arch. Br J Radiol. 2005 Oct;78(934):961-6.

11. Barranhas AD, Indiani JM, Marchiori E, Santos AA, Rochitte CE, Nacif MS. Atypical presentation of Kommerell's diverticulum. Arq Bras Cardiol 2009 Dec;93(6):e88-90.

12. Rosa P, Gillespie DL, Goff JM, O'donnell SD, Starnes B. Aberrant right subclavian artery syndrome: a case of chronic cough. J Vasc Surg. 2003 Jun;37(6):131821.

13. Carrizo GJ, Marjani MA. Dysphagia lusoria caused by an aberrant right subclavian artery. Tex Heart Inst J. 2004; 31(2):168-71.

14. Godlewski J, Widawski T, Michalak M, Kmie Z. Aneurysm of the aberrant right subclavian artery - a case report. Pol J Radiol. 2010 Oct;75(4):47-50. 